\title{
Class of two-dimensional Yang-Mills vacua and their relation to the nonlinear sigma model
}

\author{
A. Bassetto* \\ CERN, Theory Division, CH-1211 Geneva 23, Switzerland \\ and INFN, Sezione di Padova, Padua, Italy \\ G. Nardelli \\ Dipartimento di Fisica, Università di Trento, 38050 Povo (Trento), Italy \\ and INFN, Gruppo Collegato di Trento, Italy \\ (Received 23 July 1997; published 14 January 1998)
}

\begin{abstract}
Classical vacuum - pure gauge-solutions of Euclidean two-dimensional SU(2) Yang-Mills theories are studied. Topologically nontrivial vacua are found in a class of gauge group elements isomorphic to $S_{2}$. These solutions are unexpectedly related to the solution of the nonlinear $O(3)$ model and to the motion of a particle in a periodic potential. [S0556-2821(98)04304-5]

PACS number(s): 11.10.Kk, 11.10.Lm
\end{abstract}

A Coulomb gauge description of Yang-Mills fields in three spatial dimensions is ambiguous [1]: several gaugeequivalent potentials $A_{\mu}$ represent the same physical field configuration, all these potentials satisfying the Coulomb gauge and being related by large gauge transformations, i.e. gauge transformations that cannot be smoothly deformed to the identity. Consequently, although gauge-equivalent, these field configurations are topologically inequivalent. Clearly, this ambiguity persists in the pure-gauge solutions, leading to different vacua [2].

Topological arguments suggest that this pathology disappears in two spatial dimensions. Nevertheless, the situation changes when considering gauge group elements belonging to a particular class of SU(2). Topologically inequivalent vacua can indeed be found, which are related to the solutions of the non-linear $O(3)$ model on a plane and to the solutions of a particle moving in a periodic potential.

The purpose of this Brief Report is to illustrate this phenomenon.

Our conventions are as follows. Space coordinates will be denoted by $x_{\mu}, \mu=1,2 ; T^{j}=\sigma^{j} / 2 i, j=1,2,3$, will denote the anti-Hermitian SU(2) generators; the first two components of the internal indices will be denoted by $a=1,2$.

Classical vacuum solutions are pure gauges, $A_{\mu}$ $=(1 / g) U^{-1} \partial_{\mu} U$, and we shall look for non-trivial vacuum solutions satisfying the gauge choice $\partial_{\mu} A_{\mu}=0$, just as in the standard Gribov copies in three spatial dimensions. The SU(2) gauge group element $U$ will be chosen to belong to the class of transformations not depending on $\sigma^{3}$, namely

$$
U=n_{0}-i \sigma^{a} n^{a}
$$

These elements in general are not a subgroup of $\mathrm{SU}(2)$. Rather, since $n_{0}^{2}+n^{a} n^{a}=1$, they belong to a class isomorphic to $S_{2}$, which is in turn a homogeneous space of $\mathrm{SU}(2)$. The pure gauge potential associated with Eq. (1) is

\footnotetext{
* On leave of absence from Dipartimento di Fisica, Via Marzolo 8 - I-35131 Padova.
}

$$
g A_{\mu}=-2 T^{3} \epsilon^{a b} n^{a} \partial_{\mu} n^{b}+2 T^{a}\left(n_{0} \partial_{\mu} n^{a}-n^{a} \partial_{\mu} n_{0}\right)
$$

and the gauge condition $\partial_{\mu} A_{\mu}=0$ implies the following equations:

$$
\epsilon^{a b} n^{a} \Delta n^{b}=0, \quad n^{a} \Delta n_{0}-n_{0} \Delta n^{a}=0 .
$$

In turn, Eqs. (3) can be rewritten in a more compact form by renaming $n_{0} \equiv n^{3}$, so that the requirement $U \in \mathrm{SU}(2)$ in terms of $n^{i}$ becomes $n^{i} n^{i}=1, i=1,2,3$, and $\mathbf{n}=\left\{n^{i}\right\}$ satisfies the same constraint as a non-linear $O(3)$ model. Then Eqs. (3) simply become

$$
\epsilon^{i j k} n^{j} \Delta n^{k}=0
$$

Any field configuration $n^{i}$ satisfying the $O(3)$ self-duality (or anti-self-duality) conditions

$$
\partial_{\mu} n^{i}= \pm \epsilon^{i j k} \epsilon_{\mu \nu} \partial_{\nu} n^{j} n^{k}
$$

is a solution of Eqs. (4), as can be easily checked by direct inspection. It is important to stress that Eqs. (4) are not the equations of motion of the non-linear $O(3)$ model [in the non-linear $O(3)$ model the fields $n^{i}$ satisfy the equations $\left.\Delta n^{i}=n^{i} n^{j} \Delta n^{j}\right]$. Nevertheless, the same self-duality condition that solves the non-linear $O(3)$ model also solves Eqs. (4).

Any field configuration satisfying the constraint $n^{i} n^{i}=1$ has a topological invariant defined by

$$
Q=\frac{1}{8 \pi} \int d^{2} x \epsilon^{i j k} \epsilon_{\mu \nu} n^{i} \partial_{\mu} n^{j} \partial_{\nu} n^{k} \in \mathbf{Z},
$$

which is obviously the topological invariant of the non-linear $O$ (3) model as well. The integer number $Q$ simply tells how many times the stereographic projection of the Euclidean plane wraps onto the sphere $n^{i} n^{i}=1$ in the change of variables $\left\{x^{\mu}\right\} \rightarrow\left\{n^{i}\right\}$. Self-dual configurations (upper sign in Eq. (5)) have positive $Q$, whereas for anti-self-dual fields (lower sign in (5)) $Q<0$. Since the two classes of solutions are related by a parity transformation, it is not restrictive to con- 
sider only one sign in Eq. (5). For definiteness, from now on we shall discuss self-dual configurations.

The way of solving Eq. (5) is standard [3]: perform a stereographic projection of the sphere $n^{i} n^{i}=1$ onto the plane $n^{1} \times n^{2}$ and consider the stereographically projected variables $\omega^{1}=n^{1} /\left(1-n^{3}\right), \omega^{2}=n^{2} /\left(1-n^{3}\right)$. Then, Eq. (5), written in terms of the function $\omega=\omega^{1}+i \omega^{2}$, simply becomes the Cauchy-Riemann conditions, so that any meromorphic function $\omega$ is a solution. Once a meromorphic function $\omega(z)$ is chosen, the $n^{i}$ can easily be obtained by inverse stereographic projection

$$
n_{0}=n^{3}=\frac{|\omega(z)|^{2}-1}{|\omega(z)|^{2}+1}, \quad n^{a}=\frac{2 \omega^{a}}{|\omega(z)|^{2}+1} .
$$

The topological number $Q$ can be written in terms of $\omega$ as

$$
Q=\frac{1}{\pi} \int d^{2} x \frac{\left|\omega^{\prime}(z)\right|^{2}}{\left(1+|\omega(z)|^{2}\right)^{2}} .
$$

Using Eq. (2), the quantity $g^{2} A_{\mu}^{i} A_{\mu}^{i}$ can be written in the form $g^{2} A_{\mu}^{i} A_{\mu}^{i}=4 \partial_{\mu} \mathbf{n} \cdot \partial_{\mu} \mathbf{n}$. Consequently, the bilinear $g^{2} A_{\mu}^{i} A_{\mu}^{i}$ is proportional to the Lagrangian of the non-linear $O$ (3) model and, on the classical self-dual solutions (5), the topological invariant $Q$ can be simply rewritten as

$$
\begin{aligned}
\left.Q\right|_{\text {on self-dual sol. }} & =\frac{g^{2}}{32 \pi} \int d^{2} x A_{\mu}^{i} A_{\mu}^{i} \\
& =-\frac{g^{2}}{16 \pi} \int d^{2} x \operatorname{Tr}\left[A_{\mu} A_{\mu}\right] .
\end{aligned}
$$

Some remarks on Eq. (9) are in order. First of all it is not gauge invariant: as a matter of fact, "small" gauge transformations are not allowed by the gauge fixing, while "large" gauge transformations change the topological number of the vacua. Secondly, Eq. (9) cannot be taken as a definition of the topological invariant $Q$, as Eq. (9) explicitly depends on the metric. However, one should remember that expression (9) holds only when the self-duality condition (5) has been taken into account, and therefore its validity is only "on shell." It is intriguing that, "on shell," the winding number $Q$ is just a mass term for the gauge fields.

For a given $Q$, several choices of $\omega$ are possible. In turn any choice of $\omega$ determines $n_{0}, n^{a}$ through Eqs. (7). The simplest choice of meromorphic function is clearly a zero of order $k$, so that one can choose $\omega(z)=\left(z / z_{0}\right)^{k}$ where the constant $z_{0} \neq 0$ has been introduced to make $\omega$ dimensionless, as required. It is not surprising that, for such a choice of $\omega$, the topological number evaluated through Eq. (6) just gives the integer power $k$. Therefore, for a given $k$, one can always choose a pure power as the representative element of the homotopy class. This choice of $\omega$ characterizes the radially symmetric gauge group elements. Using Eqs. (7) and (1), we obtain:

$$
U_{k}=\frac{r^{2 k}-r_{0}^{2 k}}{r^{2 k}+r_{0}^{2 k}}-i \sigma^{a} E_{r}^{a} \frac{2 r_{0}^{k} r^{k}}{r_{0}^{2 k}+r^{2 k}} \equiv \cos \psi-i \sigma^{a} E_{r}^{a} \sin \psi,
$$

where $r_{0}=\left|z_{0}\right| \neq 0$ and $E_{r}^{a}$ is the radial component of the following $k-$ zweibein in polar coordinates

$$
E_{r}^{a}=(\cos k \theta, \sin k \theta), \quad E_{\theta}^{a}=(-\sin k \theta, \cos k \theta),
$$

which are nothing but the usual zweibein with angle $\theta$ replaced by $k \theta$.

It is easy to check that all the basic features of the usual polar zweibein are satisfied, i.e. they are orthonormal and cyclic under the external product: $E_{\alpha}^{a} E_{\beta}^{a}=\delta_{\alpha \beta}, E_{\alpha}^{a} E_{\alpha}^{b}=\delta^{a b}$, $\epsilon^{a b} \epsilon_{\alpha \beta} E_{\beta}^{b}=E_{\alpha}^{a}$, where $\alpha, \beta=r, \theta$ and $\epsilon^{12}=\epsilon_{r \theta}=1$.

By construction, when $\theta$ varies from 0 to $2 \pi, E_{r}^{a}$ spans $k$ times the unit circle in the Euclidean plane. Consequently, the only difference with the standard polar zweibein is in the derivatives of such vectors, which provide an extra $k$ factor, i.e.

$$
\partial_{\mu} E_{\alpha}^{a}=\frac{k}{r} \epsilon_{\alpha \beta} E_{\beta}^{a} e_{\theta}^{\mu},
$$

where we denoted by $\left(e_{r}^{\mu}, e_{\theta}^{\mu}\right)$ the standard zweibein in polar coordinates or, equivalently, the ones given in Eqs. (11) with $k=1$.

$U_{k}$ cannot be continuously deformed to the constant solution (trivial vacuum). Equation (10) with $k=1$ is identical to the gauge group element discussed by Jackiw and Rebbi in Ref. [4]. For $k>1, U_{k}$ is not equivalent to the $k$ th power of $U_{1}$; this power leads to a gauge potential that does not obey the gauge condition $\partial_{\mu} A_{\mu}=0$.

The pure gauge potential corresponding to Eq. (10) is given by

$$
\begin{gathered}
g A_{\mu}^{3}=-2 \frac{k}{r} \sin ^{2} \psi(r) e_{\theta}^{\mu}=-\frac{8 k}{r} \frac{r_{0}^{2 k} r^{2 k}}{\left(r_{0}^{2 k}+r^{2 k}\right)^{2}} e_{\theta}^{\mu}, \\
g A_{\mu}^{a} E_{r}^{a}=2 \psi^{\prime}(r) e_{r}^{\mu}=-\frac{4 k}{r} \frac{r_{0}^{k} r^{k}}{r_{0}^{2 k}+r^{2 k}} e_{r}^{\mu}, \\
g A_{\mu}^{a} E_{\theta}^{a}=\frac{k}{r} \sin 2 \psi(r) e_{\theta}^{\mu}=\frac{4 k}{r} \frac{r_{0}^{k} r^{k}\left(r^{2 k}-r_{0}^{2 k}\right)}{\left(r_{0}^{2 k}+r^{2 k}\right)^{2}} e_{\theta}^{\mu} .
\end{gathered}
$$

All the solutions (for any $k$ ) are regular.

Alternatively, one could have searched for topologically non-trivial vacua in the Abelian subgroup of $k$ radially symmetric elements of the type

$$
U_{k}=\cos \chi(r)-i \sigma^{a} E_{r}^{a} \sin \chi(r) .
$$

The gauge condition $\partial_{\mu} A_{\mu}=0$ leads to the equation

$$
\Delta(2 \chi)=\frac{k^{2}}{r^{2}} \sin 2 \chi .
$$

In turn Eq. (15) can be simplified by introducing the variables $\varphi=2 \chi$ and $\tau=k \log \left(r / r_{0}\right)$. Thus, in terms of $\varphi$ and $\tau$, eq. (15) simply becomes the one-dimensional sine-Gordon equation 


$$
\frac{d^{2} \varphi}{d \tau^{2}}=\sin \varphi
$$

describing the motion of a particle in a periodic potential $V(\varphi)=(1+\cos \varphi)$ with respect to the "time" $\tau=k \log \left(r / r_{0}\right)$.

The radially symmetric solution we have considered in Eq. (10),

$$
\chi(r)=\psi(r)=2 \tan ^{-1}\left(\frac{r_{0}}{r}\right)^{k}
$$

corresponds to selecting the initial conditions of the particle in the periodic potential in such a way that the dynamics of the system is that of a kink: the particle starts at the "time", $\tau=-\infty$ from one top of the periodic potential and reaches at $\tau=+\infty$ a contiguous top.

Another radially symmetric solution is obtained by changing the sign of $k$ in the exponent of Eq. (17). This change is equivalent to a conformal transformation and maps a kink into an anti-kink. In spite of the fact that our original system (SU(2) Yang-Mills in two Euclidean dimensions) is not a conformal model, the solutions in the class we considered are conformally invariant. Vacuum solutions are pure gauges and therefore satisfy $F_{\mu \nu}=0$. Consequently, the energymomentum tensor, in particular its trace, identically vanishes.

Starting from Eqs. (13) one can also obtain $(2+1)$-dimensional vacuum solutions simply by replacing $r_{0}$ by an arbitrary function of time, i.e. $r_{0}=r_{0}(t)$; the corresponding $g A_{0}=U^{-1} \partial_{0} U$ component of the gauge potential can be easily derived from Eq. (10). The arbitrary reparametrization of time that such solutions exhibit is not surprising. In fact vacuum solutions in $2+1$ dimensions follow from a Chern-Simons action, which is invariant under diffeomorphisms. Nevertheless the pure gauges in Eq. (13), when interpreted as $(2+1)$-dimensional solutions, exhibit reparametrization invariance only under the time component: this happens because the Coulomb gauge choice breaks diffeomorphism invariance, leaving as a residual subgroup precisely the group of time reparametrization.

Several arguments deserve consideration for future investigations.

At the classical level, one might wonder whether the interaction with matter could justify and stabilize the particular direction in the internal space to which these vacuum solutions belong. To this purpose, it seems that the most natural choices would be an interaction either with a non-linear sigma model or with a matter field in the adjoint representation; in this way the direction in the internal space of the gauge group element could be the one induced by matter. In this framework, the method developed in Ref. [5] to find classical conformal solutions of two-dimensional gauge theories interacting with matter fields seems to be the most appropriate one. Were it possible to find such a matterYang-Mills coupled system, in which a particular direction in the internal space is singled out, its vacuum structure in the vectorial sector would be identical to the solutions of the nonlinear $O(3)$ model, whose quantization is well known. Thus, the quantization of the vectorial sector would be greatly simplified; however the possible appearance of $\theta$ vacua in the complete system would depend on the dynamical properties induced by matter.

The classical coincidences we have discussed could also be relevant in the study of lower dimensional QCD effective theories. In this regard, it is intriguing that the expression for the topological invariant $Q$ on self-dual solutions we have exhibited in Eq. (9), is identical to the leading term of the Skyrmion Lagrangian.

Note added in proof. After the acceptance of this paper, we have been informed by A. Jevicki that there is a sizeable, although not complete, overlap with an investigation by Jevicki and Papanicolaou [6]. We thank Dr. Jevicki for calling our attention to his paper.

We thank Roman Jackiw for useful suggestions.
[1] V. N. Gribov, Nucl. Phys. B139, 1 (1978).

[2] R. Jackiw, I. Muzinich, and C. Rebbi, Phys. Rev. D 17, 1576 (1978); R. Jackiw, in New Frontiers in High Energy Physics, edited by B. Kursunoglu, A. Perlmutter and L. Scott (Plenum, New York, 1978).
[3] A. A. Belavin and A. M. Polyakov, JETP Lett. 22, 245 (1975).

[4] R. Jackiw and C. Rebbi, Phys. Rev. Lett. 37, 172 (1976).

[5] G. Nardelli, Phys. Rev. Lett. 73, 2524 (1994); Phys. Rev. D 52, 5944 (1995).

[6] A. Jevicki and N. Papanicolaou, Phys. Lett. 78B, 438 (1978). 